\title{
Network Analysis for the Identification of Hub Genes and Related Molecules as Potential Biomarkers Associated With the Differentiation of Bone Marrow- derived Stem Cells Into Hepatocytes
}

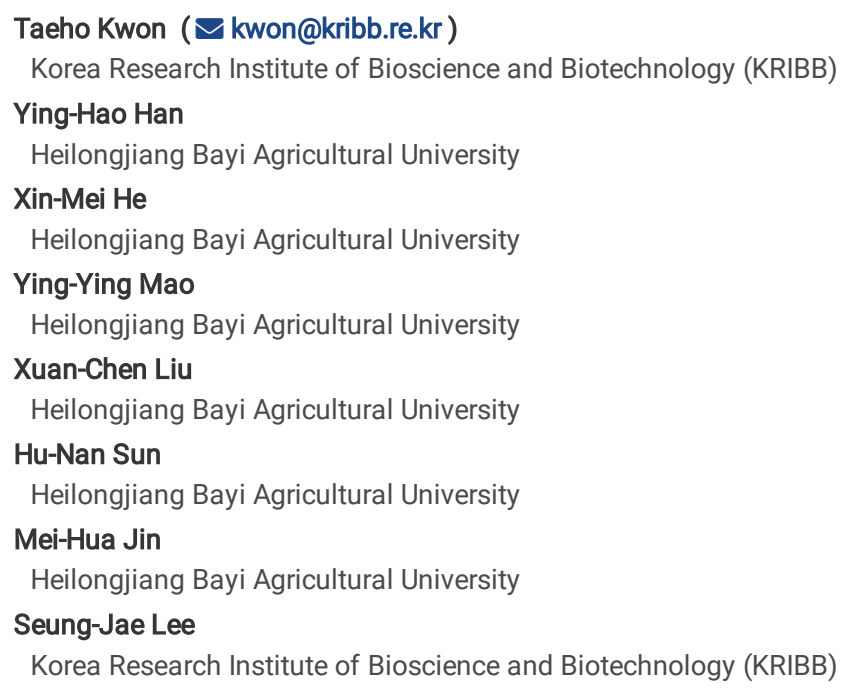




\section{Abstract}

The incidence of liver diseases has been increasing steadily. However, it has some shortcomings, such as high cost and organ donor scarcity. The application of stem cell research has brought new ideas for the treatment of liver diseases. Therefore, it is particularly important to clarify the molecular and regulatory mechanisms of differentiation of bone marrow-derived stem cells (BMSCs) into liver cells. Herein, we screened differentially expressed genes between hepatocytes and untreated BMSCs to identify the genes responsible for the differentiation of BMSCs into hepatocytes. GSE30419 gene microarray data of BMSCs and GSE72088 gene microarray data of primary hepatocytes were obtained from the Gene Expression Omnibus database. Transcriptome Analysis Console software showed that 1896 genes were upregulated and 2506 were downregulated in hepatocytes as compared with BMSCs. Hub genes were analyzed using the STRING, revealing that two hub genes, Cat and Cyp2e1, play a pivotal role in oxidation-reduction process. The results indicate that the IncRNA-miRNA-mRNA interaction chain may play an important role in the differentiation of BMSCs into hepatocytes, which provides a new therapeutic target for liver disease treatment.

\section{Introduction}

A liver transplant is a significant way to treat patients with severe liver damage, such as decompensated cirrhosis, liver failure, and advanced liver cancer. However, there is a scarcity of liver donors, and transplantation is associated with immune rejection and other problems. In recent years, with the advancement of biological treatment research, the development of molecular biology and cell biology engineering technology, and the stem cell research, stem cell therapy has emerged as an economic and feasible liver disease treatment for the end-stage liver disease, particularly decompensated cirrhosis liver failure and advanced liver cancer, and offers an effective strategy with no limit of supply and demand. Stem cell therapy has broad application prospects in liver disease. Bone marrow-derived stem cells (BMSCs) are a group of adult stem cells derived from the mesoderm. In vitro and in vivo studies have reported that BMSCs could differentiate into the cells of the mesoderm lineage, such as osteoblasts, adipocytes, muscle cells, neurons and brain cells, cardiomyocytes, and hepatocytes. Growing evidence suggests that BMSCs can differentiate into hepatocytes, presenting interesting possibilities for cellular therapy of liver diseases. Ke Z et al. reported that downregulation of Wnt signaling could promote BMSC differentiation into hepatocytes ${ }^{1}$. Krause et al. reported that BMSCs had tremendous differentiation capacity to transform into liver epithelial cells ${ }^{2}$. In addition, Kang et al. reported that rat BMSCs differentiate into hepatocytes

${ }^{3}$. Recently, a large number of studies have reported hepatocyte growth factor (HGF) and basic fibroblast growth factor (bFGF) to be the key cytokines that induce the differentiation of BMSCs into hepatocytes. Under certain conditions, HGF and bFGF can induce the differentiation of BMSCs into hepatocytes for the treatment of advanced liver disease $e^{4,5}$

Advances in RNA-sequencing techniques have led to the discovery of thousands of non-coding transcripts with unknown functions. There are several types of non-coding linear RNAs such as microRNAs (miRNA) and long non-coding RNAs (IncRNA), as well as circular RNAs (circRNAs) consisting of a closed continuous loop ${ }^{6}$. IncRNA and miRNA are the two most important types of ncRNA. miRNAs are endogenous 23-nt RNAs that pair to the mRNAs of proteincoding genes to direct their posttranscriptional repression in animals and plants ${ }^{7}$. IncRNAs are longer than $200 \mathrm{nt}^{8}$ and are characterized by low levels of sequence conservation and expression. They modulate various biological functions at epigenetic, transcriptional, and post-transcriptional levels, or directly regulate protein activity. Studies have found that IncRNA regulates miRNA in three ways: (1) as a precursor or host of miRNAs; (2) IncRNAs and miRNAs compete for mRNA binding; and (3) by absorbing some specific miRNA as a bait to regulate the expression of miRNA target genes. Furthermore, studies have shown that miRNA and IncRNA play an important role in various life activities, and in the occurrence and development of liver disease. Most importantly, these are potential therapeutic targets and diagnostic biomarkers.

At present, the mechanism underlying the differentiation of BMSCs into hepatocytes is unclear. Further investigation of miRNA and IncRNA can advance the research of the differential genes. Therefore, we identified hub genes that have significant relevance to the differentiation of BMSCs into hepatocytes along with its related miRNAs and IncRNAs including miR-692, miR-466l, miR-703, miR-137, miR-186, miR-186, miR-539, miR-23a, miR-23b, miR-466d-5p, miR-466k, Zfp469, 1700020l14Rik, Gm42418, Zfas1, Dubr and Peg13 as potential biomolecules. This study provides potential therapeutic targets for the treatment of liver diseases.

The chip data for this study were obtained from the Gene Expression Omnibus Database, the differential genes were screened by bioinformatics software, and the Gene analysis and target miRNA prediction were performed in the go-enriched protein interaction network analysis center for the differential genes.

Differential genes were screened to obtain the hub genes that control the differentiation of BMSCs into hepatocytes and liver development. Hub genes include Cat, Cyp2e1, Pah, Ugt2a3, Acss2, Aldh6a1, Hmgcs2, H6pd, Aldh1a7, Hmgcl, Ugt1a1, Arg1, Otc, Baat, Slco1b2, Onecut1, Hhex, Proc, Cdk4, Il6, Fn1, Erbb2, Ccnd1, and Bmp4.

\section{Results}

\section{DEG screening}

All the red lines in Figure 1 are on the same straight line, indicating a good degree of data standardization. A total of 4402 differential genes were detected in hepatocytes, with 1896 upregulated and 2506 downregulated genes compared with BMSCs.

\section{Principal-component analysis (PCA)}

The principal-component analysis (PCA) was performed to obtain information about the overall composition of the analyzed complex microarray datasets. It showed differences between the respective group gene expression profiles. This indicates that the group profiles are divergent and separately clustered, which 
allowed for the analysis of the differences in gene regulation. In addition, similarities of the individual values within a group are shown in Figure 2.

\section{Hierarchical cluster analysis and Volcano plot analysis of DEGs}

Hierarchical cluster analysis showed that the three BMSC samples were distributed within the BMSC sample cluster and that the three hepatocyte samples were within the hepatocyte sample cluster (Figure $3 \mathrm{~A}$ ). This showed that grouping was rational and that the data could be used directly for further analysis.

The log2-fold change difference and the negative logarithm of $p$ values between the volcano map samples of DEGs in the BMSC and hepatocyte samples are indicated on the $X$ and $Y$ axes, respectively, each point representing a single gene with detectable expression in both samples. The downregulated and upregulated genes were indicated by blue and red, respectively, and insignificant genes were indicated by gray dots. There were 1896 upregulated genes and 2506 downregulated genes in hepatocyte samples (Figure 3B).

\section{GO analysis of DEGs}

All DEGs were analyzed by DAVID software and the results of GO analysis indicated that the upregulated genes were significantly enriched in the biological processes of oxidation-reduction process (G0:0055114), lipid metabolic process (G0:0006629), metabolic process (G0:0008152), and liver development(GO:0001889) (Figure 4A); on the other hand, the downregulated genes were significantly enriched in regulation of cell cycle (G0:0007049), cell division (G0:0051301), multicellular organism development (G0:0007275), and positive regulation of cell proliferation (G0:0008284) (Figure 4B).

\section{Construction of PPI networks of oxidation-reduction process (G0:0055114), metabolic process (GO:0008152), liver development (G0:0001889), and positive regulation of cell proliferation (G0:0008284) biological process}

To screen out the hub genes during the differentiation of BMSCs into hepatocytes, upregulated genes in hepatocyte oxidation-reduction process, hepatic metabolism process and liver development, and downregulated genes in positive regulation of cell proliferation were selected because they are closely related to hepatocyte differentiation. The PPI network was constructed with four GO genes (Table 1) and STRING database. Four genes in GO terms differentially expressed genes were uploaded to the STRING for further analysis. The data exported from the STRING was screened for the hub genes. Two hub genes were involved in the oxidation-reduction process of hepatocytes: Cat and Cyp2e1. Seven genes were shown to be hub genes within the metabolic process: Pah, Ugt2a3, Acss2, Aldh6a1, Hmgcs2, H6pd, and Aldh1a7. Nine genes appeared to play an important role in liver development: namely Hmgcl, Ugt1a1, Arg1, Otc, Baat, Slco1b2, Onecut1, Hhex, and Proc; and six genes appeared to play an important role in suppressing liver differentiation: Cdk4, Il6, Fn1, Erbb2, Ccnd1 and Bmp4 (Table 2). The network was visualized and analyzed using Cytoscape v.3.8.2 software. Degree represents the number of interactions of a particular protein, and $\mathrm{BC}$ reflects the number of shortest paths through a node and is critical in analyzing the node importance. In the final network shown in Figure 5 , the nodes with the highest height are shown in a large circle, with green and pink shadows indicating the high to low BC values of the nodes.

\section{Further miRNA mining and interaction network analysis}

Twenty-four genes related to the differentiation of BMSCs into hepatocytes were screened out, and gene-miRNA analysis was performed with miRWalk software. The intersection of miRNA results predicted by TargetScan, miRDB, miRWalk, and RNA22 databases was selected as the prediction result. The selection conditions were set as $P<0.05$, the minimum seed sequence length of 7 mer, and the target gene binding region of 3 ' UTR ${ }^{9}$. Cytoscape v.3.8.2 was used to draw the interaction network as shown in Figure 6. MiRNAs with high number of gene cross-links $(\geq 2)$ were selected (Table 3).

\section{IncRNA prediction}

The corresponding IncRNAs of miR-692, miR-466I, miR-703, miR-137, miR-186, miR-383, miR-539, miR-23a, miR-23b, miR-466d-5p, and miR-466k were predicted with StarBase 2.0. The selection threshold was the highest reliability (very high stringency $\geq 3$ ). After cross-linking, six IncRNAs targeting six key miRNAs were found, including miR-23a-3p, miR-23b-3p, miR-137-3p, miR-186-5p, miR-466l-3p, and miR-539-5p. Moreover, Zfp469 was observed to target two key miRNAs (Figure7 and Table 4).

\section{Discussion}

Liver disease is a serious hazard to human health. However, the current situation of clinical intervention of liver disease is not ideal, and there is a lack of effective drugs and methods to intervene liver disease. The liver transplantation is a conventional and effective intervention method. However, transplantation cannot meet the clinical needs due to the shortage of high-quality liver cells, allograft rejection, and other problems ${ }^{10}$. Therefore, there is an urgent need to develop new methods for liver disease intervention. Currently, stem cell-based cell replacement therapy has attracted worldwide attention. BMSC transplantation provides a new way to intervene liver disease. Several studies have shown that transplanted BMSCs can differentiate into hepatocytes to replace the function of damaged hepatocytes and tissues in liver due to their directional differentiation ability, promoting the recovery of liver injury. However, the mechanism of differentiation of BMSCs into hepatocytes remains unclear. Therefore, it is of great significance to study the key genes and downstream regulatory mechanisms associated with the differentiation of BMSCs into liver cells, which can provide potential targets for BMSC-based treatment of liver failure. 
Transcriptome Analysis Console software was used to compare the differential gene expression data of BMSCs and hepatocytes. A total of 4402 differential genes were identified, of which 1896 genes were upregulated and 2506 genes were downregulated. GO enrichment analysis showed that among the upregulated genes, $11.44 \%$ were related to oxidation-reduction process, $7.16 \%$ to liver metabolism process, and $1.92 \%$ were to liver development process. Among the downregulated genes, 5.04\% were related to cell proliferation. Oxidation-reduction process, metabolism process, liver development process, and cell proliferation are closely related to differentiation of hepatocytes.

The STRING search tool was used to build PPI network, and hub genes were screened out. The hub genes Cat and Cyp2e1 are related to the oxidationreduction process of hepatocytes; Cat and FOXO3 are positively correlated with the differentiation of BMSCs into cells of the osteogenic lineage ${ }^{11}$. Cyp2 1 is expressed in the later stage of the differentiation process of mouse embryonic stem cells to hepatocytes ${ }^{12}$. The hub genes Pah, Ugt2a3, Acss2, Aldh6a1, Hmgcs2, H6pd, and Aldh1a7 are related to liver metabolism. The mRNA level of Hmgcs2 increase during the differentiation of human embryonic stem cells into hepatocellular-like cells ${ }^{13}$. The role of Pah, Ugt2a3, Acss2, Aldh6a1, H6pd, or Aldh1a7 in hepatocyte cells is currently unclear. The hub genes Hmgcl, Ugt1a1, Arg1, Otc, Baat, Slco1b2, Onecut1, Hhex, and Proc are related to liver development. Ugt1a1 is a marker of hepatocytes, and its significant expression during the differentiation of human hematopoietic stem cells (HSCs) into hepatocytes indicates that HSCs have successfully differentiated into normal hepatocytes ${ }^{14}$. Arg1 expression is observed during hepatic-like phenotype differentiation of unrestricted somatic stem cells from human umbilical cord blood in vitro ${ }^{15}$. In a study, the 201B7 cells were cultured in Hepatocyte differentiation initiating medium (HDI) for 2 days, and it was observed that the expression of hepatocellular specific transcription factors was upregulated and that of Otc was increased ${ }^{16}$. Onecut interacts with Lmx1a to promote the differentiation of ventral midbrain neural stem cells into dopamine neurons through the Wnt1-Lmx1a pathway ${ }^{17}$. Hhex regulates endoderm differentiation of embryonic stem cell derived cells into hepatocytes ${ }^{18}$. The role of $\mathrm{Hmgcl}$, Baat, Slco1b2, or Proc in hepatocyte differentiation and development is unclear. The hub genes Cdk4, II6, Fn1, Erbb2, Ccnd1, and Bmp4 regulate cell proliferation networks and are downregulated in hepatocytes compared with untreated BMSCs, suggesting inhibitory effects on liver differentiation. Studies have shown that expression of Cyclin B1 and Cdk4 during the hepatic differentiation of liver epithelial progenitor cells (LEPCs) induced by sodium butyrate may be related to the growth arrest of LEPCs shortly after treatment ${ }^{19}$. Transplantation of MSCs promotes cell proliferation and organ repair, and activation of II-6/gp130-mediated STAT3 signaling pathway via soluble IL-6 receptor is crucial in hepatic differentiation of $\mathrm{MSCs}^{20}$. Ccnd1 has been reported to be associated with liver regeneration, and it is speculated that they play a key role in mouse hepatocytes ${ }^{21}$. Ccnd1 silencing suppresses liver cancer stem cells (LCSCs) differentiation ${ }^{22}$. Bmp4 is an important regulator of cell proliferation and differentiation. Studies have shown that Bmp4 is a key cytokine for the development of mouse embryonic stem cells into hepatocytes ${ }^{23}$. Fn1 and Erbb2 have not been reported to regulate the differentiation of BMSCs into hepatocytes.

We used miRWalk to predict upstream target miRNAs that regulate gene expression. In our study, we observed 11 miRNAs that target minimum 2 genes and are involved in the differentiation of BMSCs into hepatocytes. Previous studies have reported that IncRNAs upstream of miRNAs play an important role in BMSC differentiation, so we used StarBase 2.0 for prediction. However, only miRNA-23a, miRNA-23b, miR-137, miRNA-186, miRNA-466l, and miRNA-539 can predict the corresponding upstream IncRNAs among the selected 11 miRNAs. Six IncRNAs, Zfp469, 1700020I14Rik, Gm42418, Zfas1, Dubr, and Peg13 were predicted by StarBase v2.0. Therefore, we introduced the above 6 miRNAs and their predicted IncRNAs in the regulation of BMSC differentiation. Studies have shown that CXCL13 promotes osteogenic differentiation of BMSCs by inhibiting miR-23a expression ${ }^{24}$. MiR-23b-3p upregulation facilitates the hepatic transdifferentiation of $\mathrm{MSCs}^{25}$. MiR-23b-3p overexpression promoted the differentiation of BMSCs into Type II pneumocytes and alleviated ALI in lung tissues ${ }^{26}$. In addition, silencing of miR-137-3p was found to facilitate osteogenic differentiation of BMSCs ${ }^{27}$. Current research shows that the relationship between miRNA186-5p, miRNA-466I-3p, miRNA-539-5p, and BMSCs differentiation is not very clear. Among the 6 IncRNAs, only Zfas1 was found to be associated with BMSC differentiation, while the relationship between Zfp469, 1700020114Rik, Gm42418, Dubr, Peg13, and BMSC differentiation remains unclear. Zfas1 has been reported to affect the osteogenic and adipogenic differentiation of BMSCs by sponging miR-499 thereby upregulating ephrin type-A receptor 5 (EPHA5).

However, further research is needed to confirm these results ${ }^{28}$.

Taken together, through bioinformatics analysis, we identified key genes that regulate the differentiation of BMSCs into hepatocytes and their upstream miRNAs and IncRNAs, providing potential targets for stem-cell replacement therapy for liver diseases. Therefore, it is of great significance to clarify the key genes and downstream regulation mechanism of the differentiation of BMSCs into hepatocytes.

\section{Materials And Methods Data collection}

We downloaded the gene expression profile from the Gene Expression Omnibus (GEO) database (http://www.ncbi.nlm.nih.gov/geo) ${ }^{29}$. The gene expression profiles of GSE30419 and GSE72088 were obtained from the GEO database datasets. GSE30419 included three untreated Mus musculus BMSC samples (GSM795638, GSM795639, and GSM795640), and dataset GSE72088 included three PBS treated Mus musculus primary hepatocytes samples (GSM1375704, GSM1375705, and GSM1375706).

\section{Screening of differentially expressed genes and hierarchical cluster analysis}

The differentially expressed genes (DEGs) between primary hepatocytes and BMSCs were analyzed using Transcriptome Analysis Console software. Genes with fold-changes $|\log 2|>4$ were selected as DEGs, and $P$ values $<0.05$ and a false discovery rate (FDR) of $<0.05$ were selected as thresholds.

\section{Functional enrichment analysis}


To investigate the functions of these gene signatures, we performed GO enrichment analysis. The DAVID gene annotation tool was used for GO enrichment analysis in DEG functions ${ }^{30}$. Values of $P<0.05$ and FDR $<0.05$ were used as cutoff criteria. GO terms were displayed as a significant network using Cytoscape v. 3.8.2 software ${ }^{31}$. DEGs associated with liver differentiation were clustered in liver development and regulation of cell proliferation modules.

\section{PPI network construction and the identification of hub genes}

To investigate the interaction among those genes and reveal the hub nodes in the regulation network, we constructed the PPI network using Cytoscape v. 3.8.2 software. To assess PPI information, we used Search Tool for the Retrieval of Interacting Genes Database (STRING) (https://www.string-db.org/) ${ }^{32}$. The means of the degrees were calculated to screen out genes that showed a greater degree than the mean value. The hub genes with Betweenness Centrality (BC) value greater than 0.05 and degree greater than the mean were selected.

\section{Prediction of pivotal miRNAs and construction of gene-miRNA interaction network analysis}

Genes related to the essential pathways were selected and performed with miRWalk (http://zmf.umm.uni-

heidelberg.de/apps/zmf/mirwalk/predictedmirnagene.html) to predict its targeted miRNAs ${ }^{33}$. To verify the accuracy of the results, four databases including TargetScan, miRDB, miRWalk and RNA22 were used for intersection. The final result obtained from the intersection was further processed with Cytoscape $v$ 3.8.2. Therefore, miRNAs that targeted more than two genes were selected.

\section{miRNAs-IncRNA prediction}

StarBase v2.0 tool was used to predict the upstream molecules IncRNAs of the selected miRNAs ${ }^{34}$, and the obtained data were processed using Cytoscape $v$ 3.8.2. To identify relevant IncRNAs, the intersection of predicted results of each miRNA was obtained by the cross-linked graph.

\section{Declarations}

\section{Acknowledgments}

This research was supported by Basic Science Research Program through the National Research Foundation of Korea (NRF) funded by the Ministry of Education (2020R1I1A2052417), KRIBB-RBM0112011. This work was supported by the Natural Science Foundation of Heilongjiang Province of China (LH2021C061)

\section{Author contributions}

$\mathrm{YHH}, \mathrm{XMH}$ and TK contributed to the conception of the study, writing the manuscript and performing the literature search. YYM, XCL and SJL performed the data analysis. $\mathrm{YHH}, \mathrm{MHJ}$ and HNS performed analysis and the quality assessment of the study. YHH and TK designed the experiments, supervised the research and prepared the manuscript. All authors read and approved the final manuscript.

\section{Competing interests}

The author declare that they have no competing interests.

\section{References}

1. Ke, Z. et al. Down-regulation of Wnt signaling could promote bone marrow-derived mesenchymal stem cells to differentiate into hepatocytes. Biochem Biophys Res Commun 367, 342-348, doi:10.1016/j.bbrc.2007.12.134 (2008).

2. Krause, D. S. et al. Multi-organ, multi-lineage engraftment by a single bone marrow-derived stem cell. Cell 105, 369-377 (2001).

3. Kang, X.-Q. et al. Rat bone marrow mesenchymal stem cells differentiate into hepatocytes in vitro. World J Gastroentero/ 11, 3479-3484 (2005).

4. Xie, J.-m., Chen, J.-f., Gao, Y. \& Yao, K.-h. [Hepatocyte growth factor and fibroblast growth factor-4-induced differentiation of human bone marrow mesenchymal stem cells into hepatocyte-like cells in vitro]. Nan Fang Yi Ke Da Xue Xue Bao 26, 1439-1442 (2006).

5. Tan, Y. et al. VEGF(165) expressing bone marrow mesenchymal stem cells differentiate into hepatocytes under HGF and EGF induction in vitro. Cytotechnology 64, 635-647, doi:10.1007/s10616-012-9439-0 (2012).

6. Beermann, J., Piccoli, M.-T., Viereck, J. \& Thum, T. Non-coding RNAs in Development and Disease: Background, Mechanisms, and Therapeutic Approaches. Physiol Rev 96, 1297-1325, doi:10.1152/physrev.00041.2015 (2016).

7. Bartel, D. P. MicroRNAs: target recognition and regulatory functions. Cell 136, 215-233, doi:10.1016/j.cell.2009.01.002 (2009).

8. Choi, S.-W., Kim, H.-W. \& Nam, J.-W. The small peptide world in long noncoding RNAs. Brief Bioinform 20, 1853-1864, doi:10.1093/bib/bby055 (2019). 
9. Yi, X.-H., Zhang, B., Fu, Y.-R. \& Yi, Z.-J. STAT1 and its related molecules as potential biomarkers in Mycobacterium tuberculosis infection. J Cell Mol Med 24, 2866-2878, doi:10.1111/jcmm.14856 (2020).

10. Hang, H.-L. \& Xia, Q. Role of BMSCs in liver regeneration and metastasis after hepatectomy. World J Gastroentero/ 20, 126-132, doi:10.3748/wjg.v20.i1.126 (2014).

11. Wu, J. et al. Identification of potential specific biomarkers and key signaling pathways between osteogenic and adipogenic differentiation of hBMSCs for osteoporosis therapy. J Orthop Surg Res 15, 437, doi:10.1186/s13018-020-01965-3 (2020).

12. Maezawa, K. et al. Expression of cytochrome P450 and transcription factors during in vitro differentiation of mouse embryonic stem cells into hepatocytes. Drug Metab Pharmacokinet 23, 188-195 (2008).

13. Zamule, S. M., Coslo, D. M., Chen, F. \& Omiecinski, C. J. Differentiation of human embryonic stem cells along a hepatic lineage. Chem Biol Interact 190 , 62-72, doi:10.1016/j.cbi.2011.01.009 (2011).

14. Kumar, P. S., Chandrasekhar, C., Srikanth, L. \& Sarma, P. V. G. K. Down-regulation of SOX17, GATA4 and FoxA2 promotes differentiation potential of hepatocytes from human hematopoietic stem cells. Tissue Cell 62, 101312, doi:10.1016/j.tice.2019.101312 (2020).

15. Waclawczyk, S., Buchheiser, A., Flögel, U., Radke, T. F. \& Kögler, G. In vitro differentiation of unrestricted somatic stem cells into functional hepatic-like cells displaying a hepatocyte-like glucose metabolism. Journal of cellular physiology 225, 545-554, doi:10.1002/jcp.22237 (2010).

16. Tomizawa, M. et al. An Optimal Medium Supplementation Regimen for Initiation of Hepatocyte Differentiation in Human Induced Pluripotent Stem Cells. J Cell Biochem 116, 1479-1489, doi:10.1002/jcb.25139 (2015).

17. Yuan, J., Lei, Z.-n., Wang, X., Deng, Y.-J. \& Chen, D.-B. Interaction between Oc-1 and Lmx1a promotes ventral midbrain dopamine neural stem cells differentiation into dopamine neurons. Brain research 1608, 40-50, doi:10.1016/j.brainres.2015.02.046 (2015).

18. Kubo, A. et al. The homeobox gene Hex regulates hepatocyte differentiation from embryonic stem cell-derived endoderm. Hepatology 51, 633-641, doi:10.1002/hep.23293 (2010).

19. Li, W. et al. Hepatic differentiation and transcriptional profile of the mouse liver epithelial progenitor cells (LEPCs) under the induction of sodium butyrate. Front Biosci 12, 1691-1698 (2007).

20. Lam, S. P. et al. Activation of interleukin-6-induced glycoprotein 130/signal transducer and activator of transcription 3 pathway in mesenchymal stem cells enhances hepatic differentiation, proliferation, and liver regeneration. Liver Transp/ 16, 1195-1206, doi:10.1002/It.22136 (2010).

21. Anderson, S. P. et al. Delayed liver regeneration in peroxisome proliferator-activated receptor-alpha-null mice. Hepatology 36, 544-554 (2002).

22. Ding, H., Wang, Y. \& Zhang, H. CCND1 silencing suppresses liver cancer stem cell differentiation and overcomes 5-Fluorouracil resistance in hepatocellular carcinoma. J Pharmacol Sci 143, 219-225, doi:10.1016/j.jphs.2020.04.006 (2020).

23. Tanaka, H. et al. Chondrogenic differentiation of murine embryonic stem cells: effects of culture conditions and dexamethasone. J Cell Biochem $93,454-$ 462 (2004).

24. Tian, F., Ji, X.-L., Xiao, W.-A., Wang, B. \& Wang, F. CXCL13 Promotes Osteogenic Differentiation of Mesenchymal Stem Cells by Inhibiting miR-23a Expression. Stem cells international 2015, 632305, doi:10.1155/2015/632305 (2015).

25. Raut, A. \& Khanna, A. Enhanced expression of hepatocyte-specific microRNAs in valproic acid mediated hepatic trans-differentiation of human umbilical cord derived mesenchymal stem cells. Exp Cell Res 343, 237-247, doi:10.1016/j.yexcr.2016.03.015 (2016).

26. Zhang, P., Liu, L., Yao, L. \& Song, X. Improved Differentiation Ability and Therapeutic Effect of miR-23a-3p Expressing Bone Marrow-Derived Mesenchymal Stem Cells in Mice Model with Acute Lung Injury. Int J Stem Cells 14, 229-239, doi:10.15283/ijsc20136 (2021).

27. Kong, L. et al. Silencing MicroRNA-137-3p, which Targets RUNX2 and CXCL12 Prevents Steroid-induced Osteonecrosis of the Femoral Head by Facilitating Osteogenesis and Angiogenesis. Int J Biol Sci 16, 655-670, doi:10.7150/ijbs.38713 (2020).

28. Wu, J. et al. Long noncoding RNA ZFAS1 suppresses osteogenic differentiation of bone marrow-derived mesenchymal stem cells by upregulating miR499-EPHA5 axis. Mol Cell Endocrinol 539, 111490, doi:10.1016/j.mce.2021.111490 (2021).

29. Clough, E. \& Barrett, T. The Gene Expression Omnibus Database. Methods Mol Biol 1418, doi:10.1007/978-1-4939-3578-9_5 (2016).

30. Dennis, G. et al. DAVID: Database for Annotation, Visualization, and Integrated Discovery. Genome Bio/ 4, P3 (2003).

31. Shannon, P. et al. Cytoscape: a software environment for integrated models of biomolecular interaction networks. Genome Res 13, 2498-2504 (2003).

32. Szklarczyk, D. et al. STRING v11: protein-protein association networks with increased coverage, supporting functional discovery in genome-wide experimental datasets. Nucleic Acids Res 47, D607-D613, doi:10.1093/nar/gky1131 (2019).

33. Sticht, C., De La Torre, C., Parveen, A. \& Gretz, N. miRWalk: An online resource for prediction of microRNA binding sites. PLoS One 13, e0206239, doi:10.1371/journal.pone.0206239 (2018).

34. Li, J.-H., Liu, S., Zhou, H., Qu, L.-H. \& Yang, J.-H. starBase v2.0: decoding miRNA-ceRNA, miRNA-ncRNA and protein-RNA interaction networks from largescale CLIP-Seq data. Nucleic Acids Res 42, D92-D97, doi:10.1093/nar/gkt1248 (2014).

\section{Tables}

Table 1

Genes in $\mathrm{GO}$ terms of liver development and regulation of cell proliferation 


\begin{tabular}{|c|c|c|}
\hline GO terms & $\begin{array}{l}\text { Biological } \\
\text { process }\end{array}$ & Genes \\
\hline GO:0055114 & $\begin{array}{l}\text { oxidation- } \\
\text { reduction } \\
\text { process }\end{array}$ & $\begin{array}{l}\text { DMGDH/ALDH1L1/STEAP4/GLDC/CYP3A11/CYP3A13/H2- } \\
\text { KE6/MSM01/CMAH/TM7SF2/CYP2D9/CYP2D10/TD02/FADS6/IYD/PHYHD1/CYP2C70/ACAD11/PHYH/HGD/DIO1/DHDI }\end{array}$ \\
\hline G0:0008152 & $\begin{array}{l}\text { metabolic } \\
\text { process }\end{array}$ & ALDH1L1/ ACSM3/ ACSM1/ ACSM5/ SCP2/ ENPP2/ ACAD11/ ACSL1/ ACSL4/ HOGA1/ UPB1/ AMY1/ SLC27A2/ ALDH7A \\
\hline G0:0001889 & $\begin{array}{l}\text { liver } \\
\text { development }\end{array}$ & CEBPA/ONECUT2/ONECUT1/HP/PCSK9/AK4/AHR/HMGCL/HHEX/SLC01B2/SLC02B1/GNPNAT1/ASL/ACADM/UGT1A1/C \\
\hline GO:0008284 & $\begin{array}{l}\text { Positive } \\
\text { regulation of } \\
\text { cell } \\
\text { proliferation }\end{array}$ & CSF1/CDCA7L/RTKN2/TNC/FOXM1/RBPJ/ETS1/CTGF/CDC20/MECP2/LGALS3/FGF7/CCND2/EFEMP1/CCND1/PLAU/HC \\
\hline
\end{tabular}

Table 2

Hub genes selected based on visualize parameters like $\mathrm{BC}$ and degree

\begin{tabular}{|c|c|c|c|c|}
\hline Network name & Gene & Degree & $\mathrm{BC}$ & $A D$ \\
\hline \multirow[t]{2}{*}{ oxidation-reduction process } & Cat & 40 & 0.119805098 & \multirow[t]{2}{*}{17.90} \\
\hline & Сyp2e1 & 50 & 0.062124918 & \\
\hline \multirow[t]{7}{*}{ metabolic process } & Pah & 17 & 0.070184791 & \multirow[t]{7}{*}{10.37} \\
\hline & Ugt2a3 & 15 & 0.065342994 & \\
\hline & Acss2 & 20 & 0.060943054 & \\
\hline & Aldh6a1 & 18 & 0.059708481 & \\
\hline & Hmgcs2 & 20 & 0.053147572 & \\
\hline & H6pd & 21 & 0.119700331 & \\
\hline & Aldh1a7 & 11 & 0.069738078 & \\
\hline \multirow[t]{9}{*}{ liver development } & $\mathrm{Hmgcl}$ & 3 & 0.257142857 & \multirow[t]{9}{*}{2.57} \\
\hline & Ugt1a1 & 4 & 0.257142857 & \\
\hline & Arg1 & 3 & 0.133333333 & \\
\hline & Otc & 5 & 0.393650794 & \\
\hline & Baat & 4 & 0.4 & \\
\hline & Slco1b2 & 5 & 0.320634921 & \\
\hline & Onecut1 & 3 & 0.5 & \\
\hline & Hhex & 3 & 0.5 & \\
\hline & Proc & 4 & 0.215873016 & \\
\hline \multirow[t]{6}{*}{ positive regulation of cell proliferation } & Cdk4 & 14 & 0.107299978 & \multirow[t]{6}{*}{10.59} \\
\hline & 116 & 34 & 0.145058759 & \\
\hline & Fn1 & 38 & 0.176673936 & \\
\hline & Erbb2 & 27 & 0.076760508 & \\
\hline & Ccnd1 & 31 & 0.180939463 & \\
\hline & Bmp4 & 28 & 0.137307941 & \\
\hline
\end{tabular}

Table 3

Page $7 / 15$ 
miRNAs and its target genes

\begin{tabular}{|lll|}
\hline miRNA & Genes targeted By miRNA & Gene count \\
\hline miR-692 & Otc,Hmgcs2,Ccnd1 & 3 \\
\hline miR-466l & Bmp4,Fn1,Il6 & 3 \\
\hline miR-703 & Hhex,Hmgcl & 2 \\
\hline miR-137 & Otc,Pah & 2 \\
\hline miR-186 & Otc,Ccnd1 & 2 \\
\hline miR-383 & Ugt1a1,Pah & 2 \\
\hline miR-539 & Aldh6a1,ah & 2 \\
\hline miR-23a & Pah,Ccnd1 & 2 \\
\hline miR-23b & Pah,Ccnd1 & 2 \\
\hline miR-466d-5p & Cat,Bmp4 & 2 \\
\hline miR-466k & Cat,Bmp4 & 2 \\
\hline
\end{tabular}

Table 4

IncRNAs and its target miRNAs

\begin{tabular}{|lll|}
\hline IncRNA & MiRNAs targeted By IncRNA & MiRNA count \\
\hline Zfp469 & miR-23a,miR-23b & 2 \\
1700020114Rik & miR-137 & 1 \\
\hline Gm42418 & miR-137 & 1 \\
\hline Zfas1 & miR-186 & 1 \\
Dubr & miR-466l & 1 \\
\hline Peg13 & miR-539 & 1 \\
\hline
\end{tabular}

\section{Figures}


A

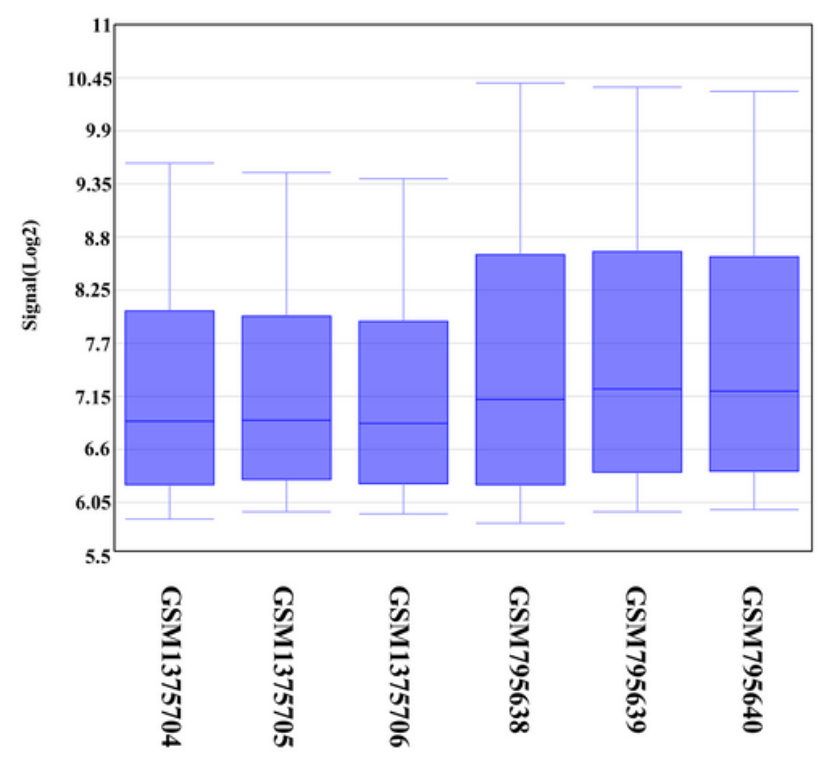

B

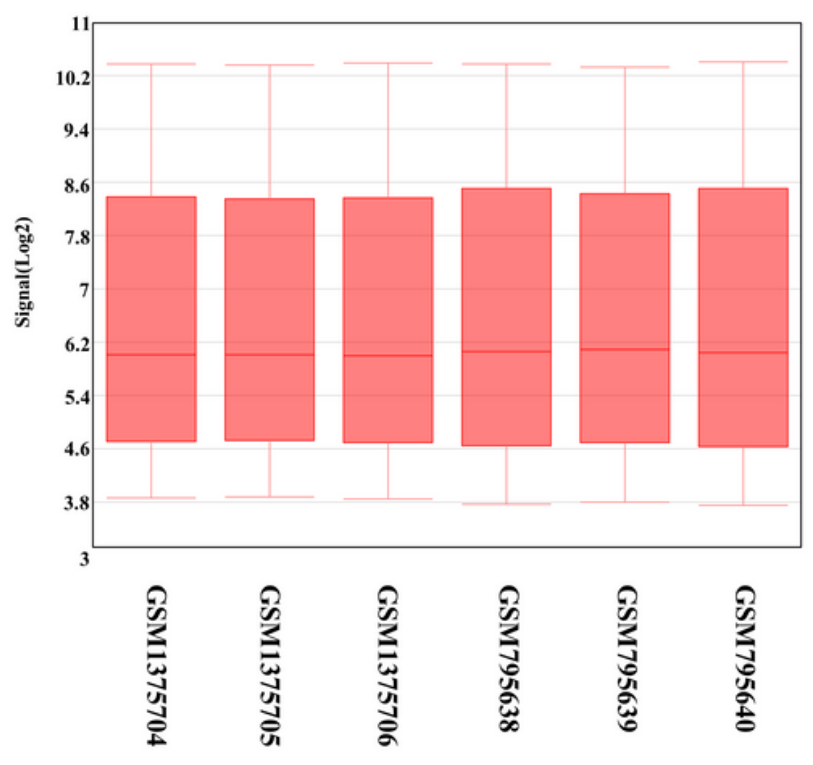

Figure 1

Cassette figures of the expression data before and after standardization

The horizontal axis represents the sample names while the vertical axis indicates the expression value. (A) Before the standardization. (B) After the standardization. 
PCA ( 98.5\%)

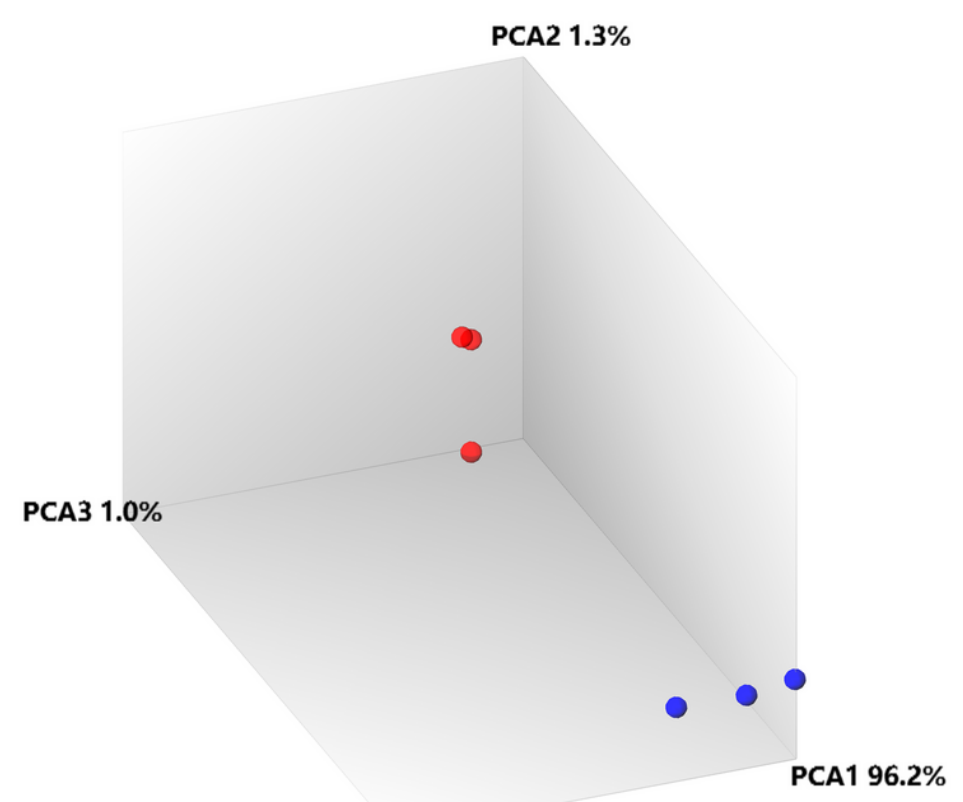

Figure 2

Principal-component analysis

PCA performed in the transcriptome of the two different sample groups. The differently colored circles indicate the two different samples. The blue circles represent the hepatocyte samples and the red circles represent the BMSC samples. Fractions of the percentage of variation out of the total mapped difference (98.5\%) among samples are indicated on each axis. 
A

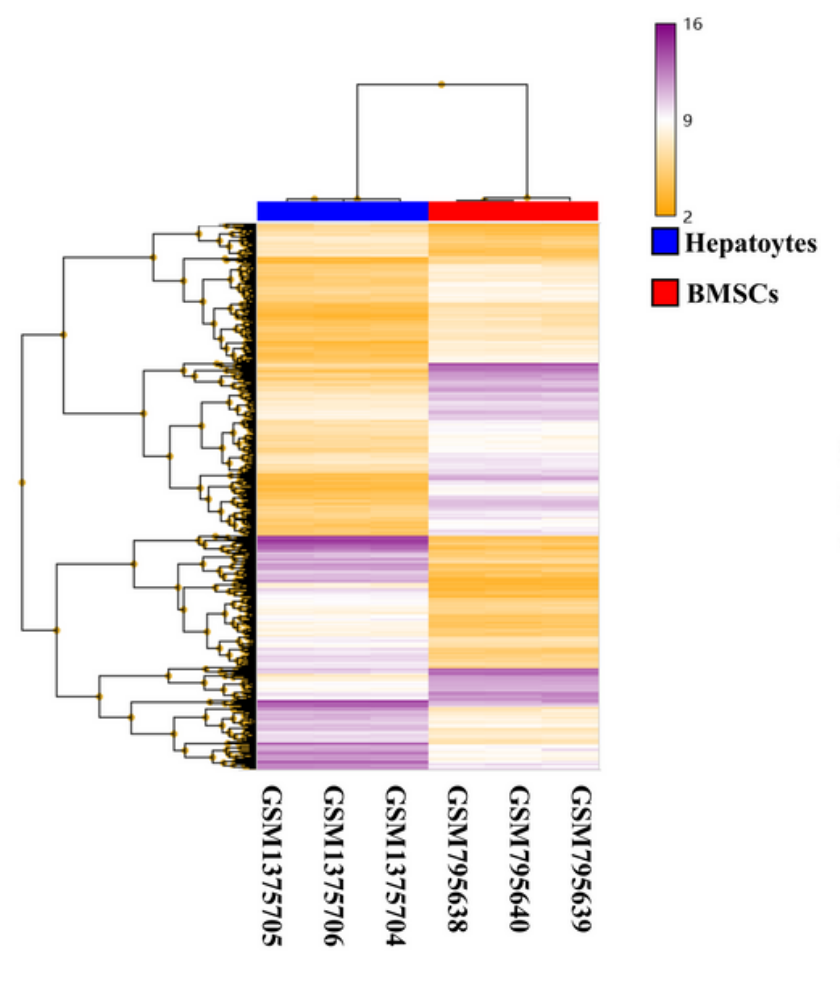

B

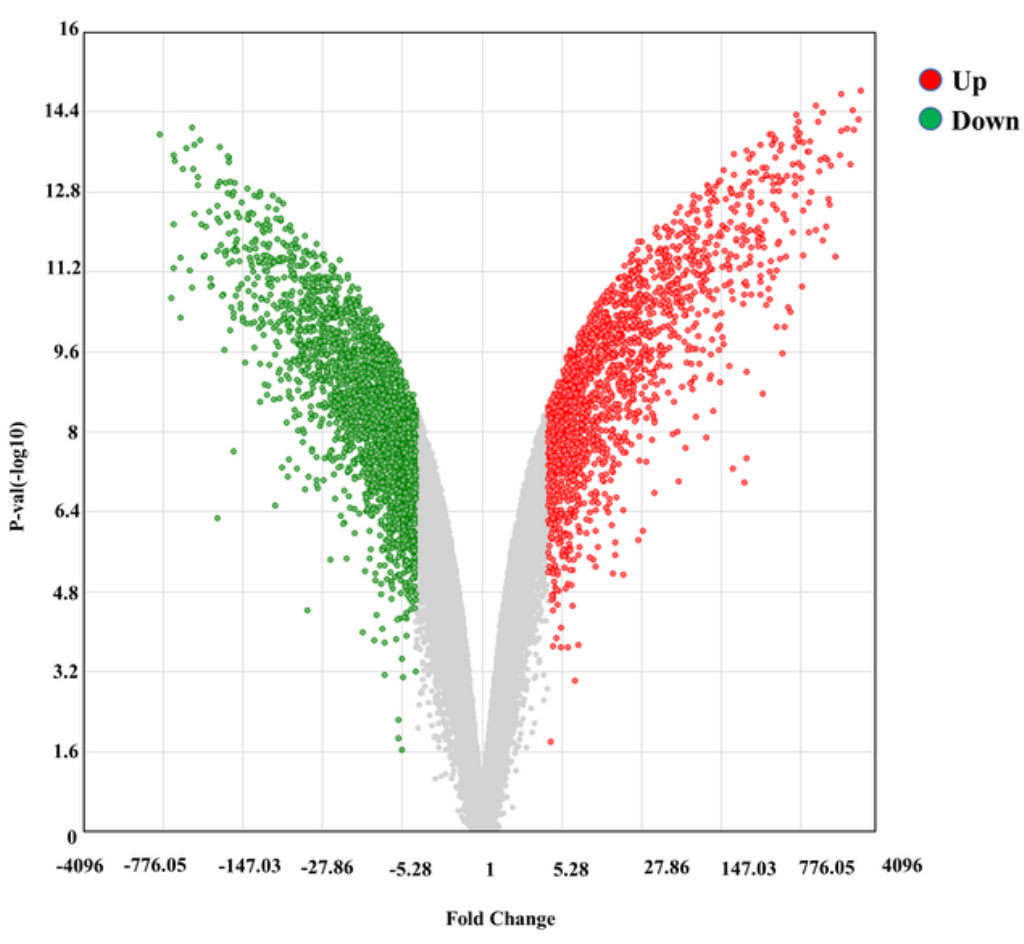

Figure 3

Hierarchical cluster and volcano plot analysis of DEGs

(A) GSM795638, GSM795639, GSM795640 are BMSC samples, and GSM1375704, GSM1375705, and GSM1375706 are hepatocyte samples. The right vertical axis represents the clustering of DEGs, and the horizontal axis represents the clustering of samples. The purple color represents the upregulated genes, and the yellow color represents the downregulated genes. (B) DEGs in the BMSC and hepatocyte samples were shown in the volcano plot, with the red dots representing significantly upregulated genes in hepatocytes samples and green dots representing significantly downregulated genes. 
A

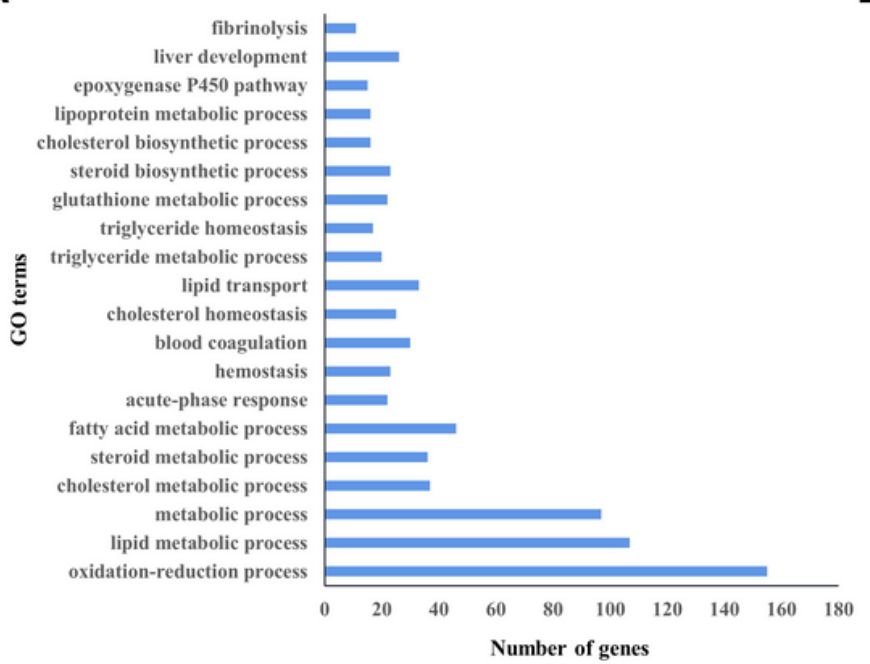

B

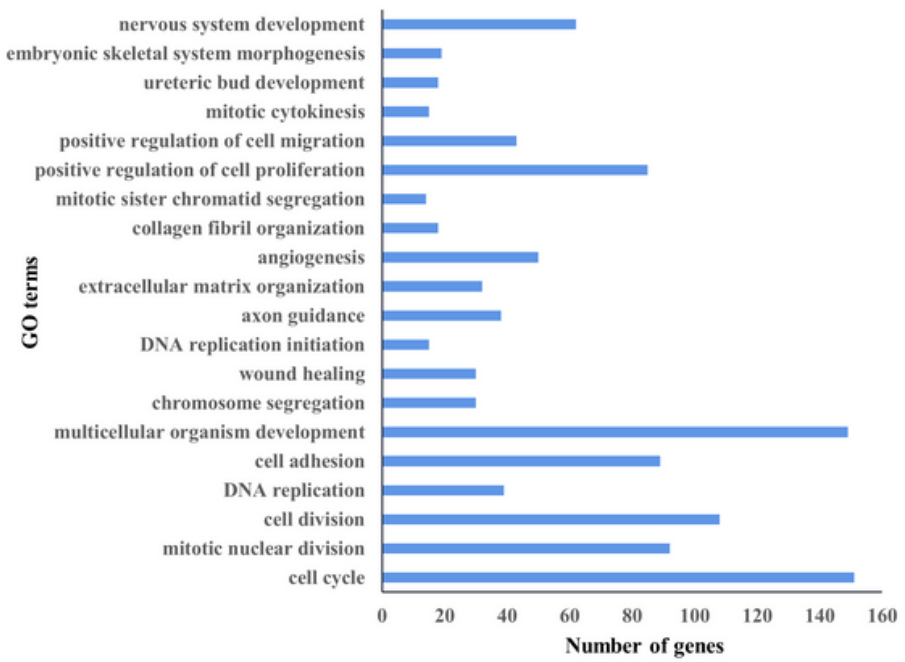

Figure 4

\section{GO enrichment analyses}

(A) The top 20 GO terms enriched for the upregulated genes of hepatocytes compared with those in case of the BMSCs. (B) The top 20 GO terms enriched for the downregulated genes of hepatocytes compared with those in case of the BMSCs.

The vertical axis represents the enriched GO terms. The horizontal axis represents the number of genes for which the GO terms were enriched. The $P$ values increase from the bottom to the top. 

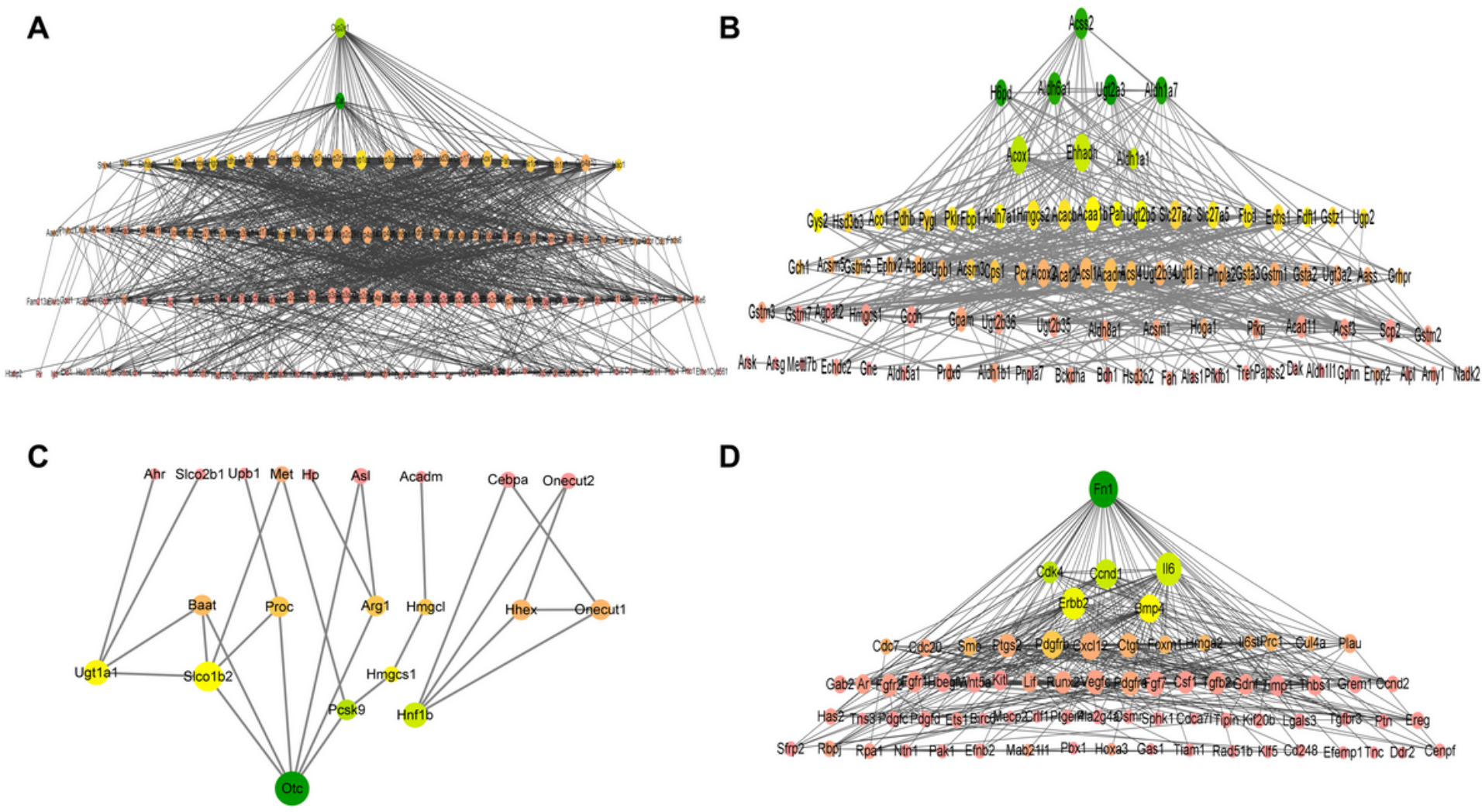

D

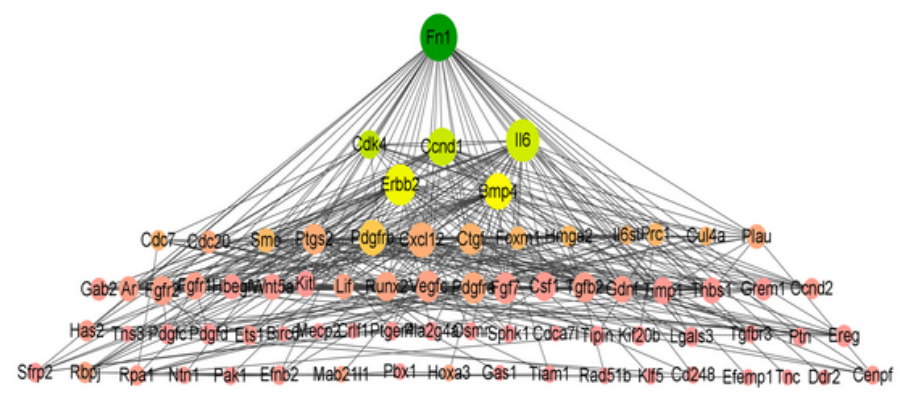

Figure 5

PPI networks of oxidation-reduction process (GO:0055114, A), metabolic process (GO:0008152, B), liver development (GO:0001889, C), and positive regulation of cell proliferation (GO:0042127, D) biological process

(A) PPI networks of oxidation-reduction process. (B) PPI networks of metabolic process. (C) PPI networks of liver development. (D) PPI networks of positive regulation of cell proliferation. Node color: shades of green to yellow color depict the nodes with the highest to lowest values of BC; Node size: sizes from the biggest to smallest circle indicate the node degrees. Bigger and dark colored nodes represent genes with more links. 


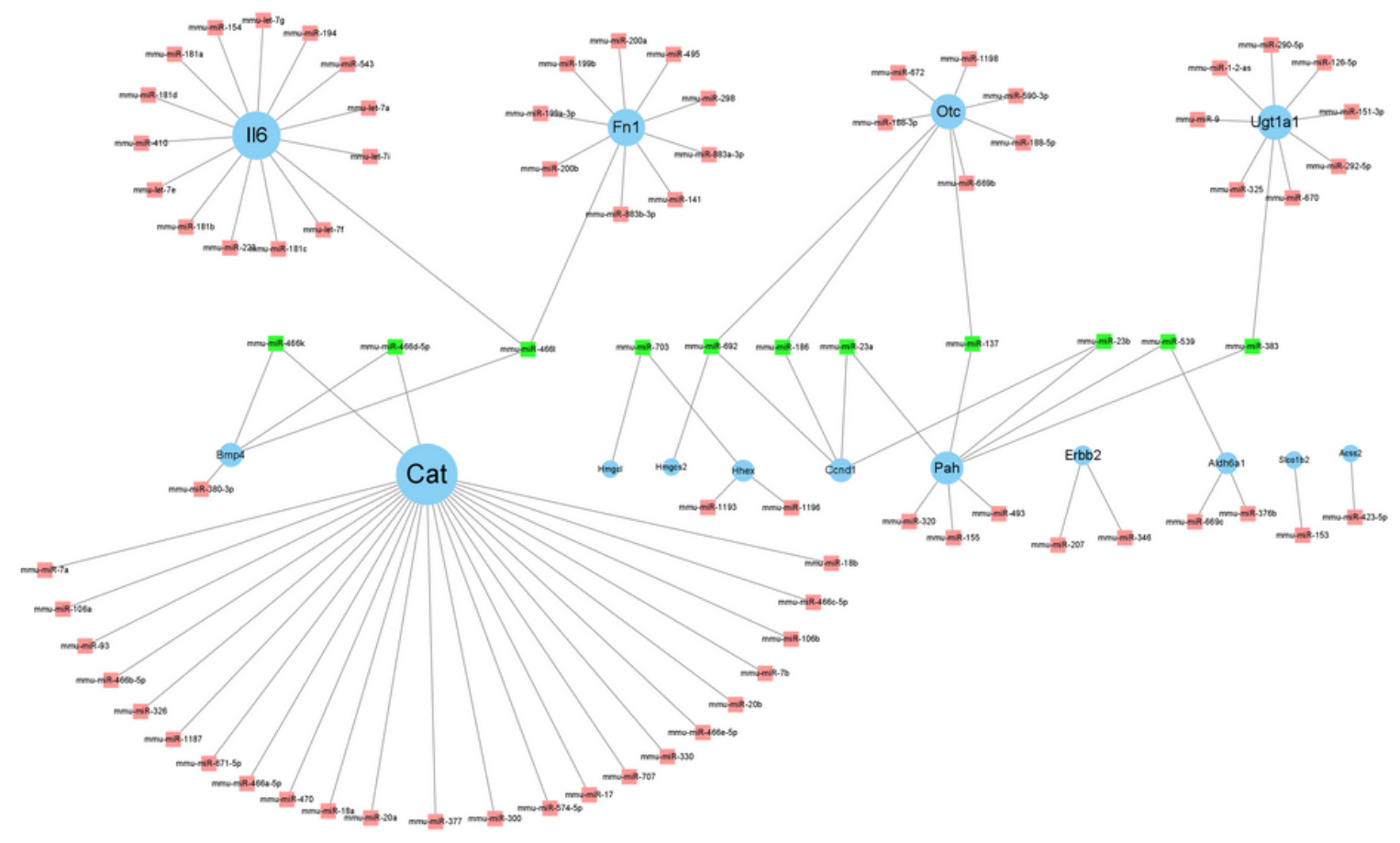

Figure 6

Interaction network between genes involved in the differentiation of BMSCs into hepatocytes and its targeted miRNAs.

Cytoscape v. 3.8.2 software was used to visualize the relationship between genes and their targeted miRNAs.

Genes are colored in blue, and node size is adjusted according to number of targeted miRNAs; miRNAs are colored in pink; miRNAs targeting more than two genes simultaneously are colored in green.
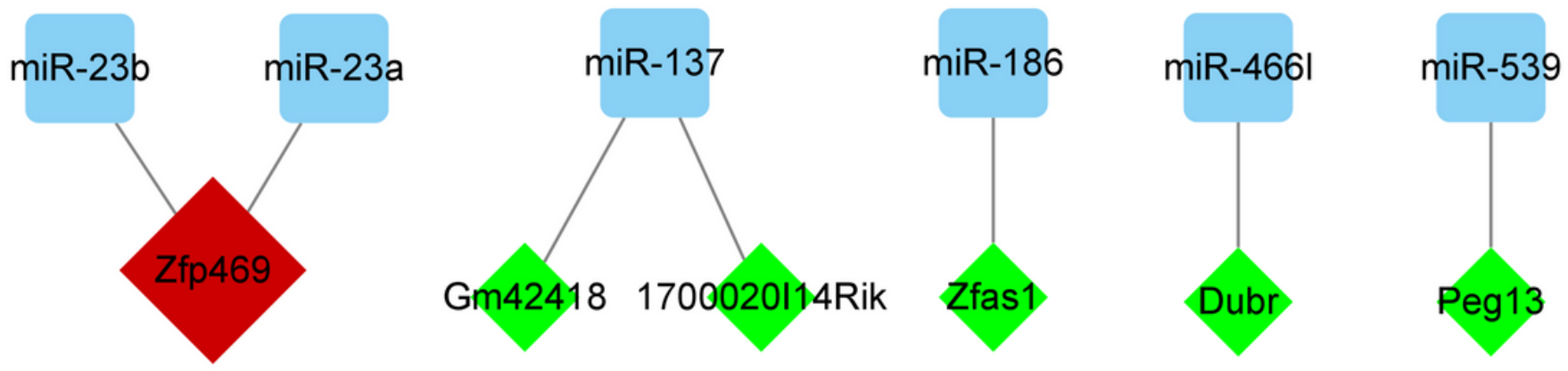

Figure 7

Interaction network between miRNAs involved in the differentiation of BMSCs into hepatocytes and its targeted IncRNAs.

(A) Use Cytoscape v. 3.8.2 software to visualize the relationship between miRNAs and its targeted IncRNAs. 
MiRNAs are colored in blue, and node size is adjusted according to number of targeted IncRNAs; IncRNAs are colored in green; miRNAs targeting more than two miRNAs simultaneously are colored in red. 